\title{
More on a SUSYQM approach to the harmonic oscillator with nonzero minimal uncertainties in position and/or momentum
}

\author{
C Quesne ${ }^{\dagger}$ and V M Tkachuk \\ † Physique Nucléaire Théorique et Physique Mathématique, Université Libre de Bruxelles, \\ Campus de la Plaine CP229, Boulevard du Triomphe, B-1050 Brussels, Belgium \\ $\ddagger$ Ivan Franko Lviv National University, Chair of Theoretical Physics, \\ 12, Drahomanov Street, Lviv UA-79005, Ukraine \\ E-mail: cquesne@ulb.ac.be and tkachuk@ktf.franko.lviv.ua
}

\begin{abstract}
We continue our previous application of supersymmetric quantum mechanical methods to eigenvalue problems in the context of some deformed canonical commutation relations leading to nonzero minimal uncertainties in position and/or momentum. Here we determine for the first time the spectrum and the eigenvectors of a onedimensional harmonic oscillator in the presence of a uniform electric field in terms of the deforming parameters $\alpha, \beta$. We establish that whenever there is a nonzero minimal uncertainty in momentum, i.e., for $\alpha \neq 0$, the correction to the harmonic oscillator eigenvalues due to the electric field is level dependent. In the opposite case, i.e., for $\alpha=0$, we recover the conventional quantum mechanical picture of an overall energy-spectrum shift even when there is a nonzero minimum uncertainty in position, i.e., for $\beta \neq 0$. Then we consider the problem of a $D$-dimensional harmonic oscillator in the case of isotropic nonzero minimal uncertainties in the position coordinates, depending on two parameters $\beta, \beta^{\prime}$. We extend our methods to deal with the corresponding radial equation in the momentum representation and rederive in a simple way both the spectrum and the momentum radial wave functions previously found by solving the differential equation. This opens the way to solving new $D$-dimensional problems.
\end{abstract}

PACS numbers: 02.30.Gp, 03.65.Fd, 11.30.Pb

Keywords: Harmonic oscillator; Electric field; Uncertainty relations; $q$-Deformations 


\section{Introduction}

During recent years, there has been much interest in studying quantum mechanical problems under the assumption of a modified Heisenberg uncertainty relation leading to nonzero minimal uncertainties in position and/or momentum. Such works are motivated by several independent lines of investigations in string theory and quantum gravity, which suggest the existence of a finite lower bound to the possible resolution of length $\Delta x_{0}$ (see, e.g., $[1,2,3])$. Furthermore, the absence of plane waves or momentum eigenvectors on generic curved spaces also hint at a finite lower bound to the possible resolution of momentum $\Delta p_{0}$ (see, e.g., [4]).

Such nonzero minimal uncertainties in position and momentum can be described in the framework of small quadratic corrections to the canonical commutation relations $[5,6,7]$. These corrections can also provide an effective description of non-pointlike particles, such as quasiparticles and various collective excitations in solids, or composite particles, such as nucleons and nuclei [6].

The resolution of quantum mechanical problems with such deformed canonical commutation relations has been mostly restricted to the case where there is only a nonzero minimal uncertainty in position. Then one may indeed consider a deformed Schrödinger equation in momentum representation and solve it using the technique of differential equations. An exact solution to the one-dimensional harmonic oscillator problem has been obtained in this way [8]. This approach has been extended to $D$ dimensions [9] and some ladder operators have been constructed [10]. Some perturbative or partial results have also been obtained for the hydrogen atom $[11,12]$.

The case where there are nonzero minimal uncertainties in both position and momentum is much more involved because there is neither position nor momentum representation, so that one has to resort to a generalized Fock space representation or, equivalently, to the corresponding Bargmann representation $[5,13]$. In this context, we have recently solved [14] the eigenvalue problem for the one-dimensional harmonic oscillator in a purely algebraic way by availing ourselves of an extension of supersymmetric quantum mechanical (SUSYQM) and shape-invariance techniques. The SUSYQM formalism [15, 16] supplemented with shape invariance under parameter translation [17, 18] is known to be a reformulation of the factorization method developed by Schrödinger [19] and by Infeld and Hull [20] (for a comparison between these two methods and corresponding references see [16, 21]). The 
procedure that we have used relies on a generalized type of shape invariance, namely that connected with parameter scaling [22, 23, 24, 25].

The purpose of the present paper is to further illustrate the power of SUSYQM techniques in solving eigenvalue problems corresponding to deformed canonical commutation relations.

First, we will consider the case of a one-dimensional harmonic oscillator with nonzero minimal uncertainties in position and momentum in the presence of a uniform electric field. Contrary to the solution of the corresponding problem in conventional quantum mechanics, which can be obtained from the solution without electric field by a simple coordinate shift, that of the present problem is more involved and will reveal some new features.

Next, we will show how to extend our method to higher-dimensional problems by providing an alternative and simple solution to the eigenvalue problem for the $D$-dimensional harmonic oscillator with isotropic nonzero minimal uncertainties in the position coordinates, which was previously dealt with by the differential equation technique [9].

Our paper is organized as follows. The one-dimensional harmonic oscillator in a uniform electric field is considered in section 2. Section 3 deals with the $D$-dimensional harmonic oscillator. Finally, section 4 contains the conclusion.

\section{One-dimensional harmonic oscillator in a uniform electric field}

Let us consider the case of a particle of mass $m$ and charge $\bar{q}$ in a harmonic potential $\frac{1}{2} m \omega^{2} x^{2}$ and a uniform electric field $\overline{\mathcal{E}}$ parallel to the $x$-axis. It is described by the Hamiltonian

$$
H=\frac{p^{2}}{2 m}+\frac{1}{2} m \omega^{2} x^{2}-\bar{q} \overline{\mathcal{E}} x
$$

and the corresponding eigenvalue problem reads

$$
H\left|\psi_{n}\right\rangle=E_{n}\left|\psi_{n}\right\rangle \quad n=0,1,2, \ldots
$$

Here $x$ and $p$ are assumed to satisfy the deformed canonical commutation relation

$$
[x, p]=\mathrm{i} \hbar\left(1+\bar{\alpha} x^{2}+\bar{\beta} p^{2}\right)
$$

where $\bar{\alpha} \geq 0, \bar{\beta} \geq 0$, and $\bar{\alpha} \bar{\beta}<\hbar^{-2}$, so that the minimal uncertainties in position and mo-

mentum are given by $\Delta x_{0}=\hbar \sqrt{\bar{\beta} /\left(1-\hbar^{2} \bar{\alpha} \bar{\beta}\right)}$ and $\Delta p_{0}=\hbar \sqrt{\bar{\alpha} /\left(1-\hbar^{2} \bar{\alpha} \bar{\beta}\right)}$, respectively [5]. 
In terms of dimensionless operators, $X=x / a, P=p a / \hbar, h=H /(\hbar \omega)$ and dimensionless parameters $\alpha=\bar{\alpha} a^{2}$ and $\beta=\bar{\beta} \hbar^{2} / a^{2}, \mathcal{E}=\bar{q} \overline{\mathcal{E}} a /(\hbar \omega)$, where $a=\sqrt{\hbar /(m \omega)}$, equations (2.1) (2.3) can be rewritten as

$$
\begin{gathered}
h=\frac{1}{2}\left(P^{2}+X^{2}\right)-\mathcal{E} X \\
h\left|\psi_{n}\right\rangle=e_{n}\left|\psi_{n}\right\rangle \quad e_{n} \equiv E_{n} /(\hbar \omega) \quad n=0,1,2, \ldots \\
{[X, P]=\mathrm{i}\left(1+\alpha X^{2}+\beta P^{2}\right)}
\end{gathered}
$$

with $\alpha \geq 0, \beta \geq 0$, and $\alpha \beta<1$.

\subsection{Energy spectrum}

At this stage, it is worth noting that going to transformed operators $X^{\prime}=X-\mathcal{E}$ and $P^{\prime}=P$, as in conventional quantum mechanics, would convert $h$ into a shifted harmonic oscillator Hamiltonian

$$
h=\frac{1}{2}\left(P^{\prime 2}+X^{\prime 2}\right)-\frac{1}{2} \mathcal{E}^{2}
$$

but, at the same time, change the commutation relation (2.6) into

$$
\left[X^{\prime}, P^{\prime}\right]=\mathrm{i}\left(1+\alpha X^{\prime 2}+\beta P^{\prime 2}+2 \alpha \mathcal{E} X^{\prime}+\alpha \mathcal{E}^{2}\right)
$$

Hence, except for $\alpha=0$, the energy spectrum of $h$ cannot be deduced from that for $\mathcal{E}=0$ obtained in our previous paper [14] (henceforth referred to as I and whose equations will be subsequently quoted by their number preceded by I).

Let us instead try to factorize $h$ as

$$
h=B^{+}(g, s, r) B^{-}(g, s, r)+\epsilon_{0}
$$

where

$$
B^{ \pm}(g, s, r)=\frac{1}{\sqrt{2}}(s X \mp \mathrm{i} g P+r)
$$

and $\epsilon_{0}$ is the factorization energy. In (2.10), $g, s$ and $r$ are assumed to be three constants depending on the parameters $\alpha, \beta, \mathcal{E}$ of the problem. The first two, $g$ and $s$, are chosen positive and going to 1 in the limit $\alpha, \beta \rightarrow 0$, while $r$ is taken as going to $-\mathcal{E}$ in the same limit. With this choice, the operators $B^{+}(g, s, r)$ and $B^{-}(g, s, r)$ will be counterparts of the creation and annihilation operators for the shifted harmonic oscillator occurring in conventional quantum mechanics. 
On inserting (2.10) in (2.9) and comparing the resulting expression with (2.4), we get four conditions, of which the first two are independent of $\mathcal{E}$ and coincide with equations (I2.7) and (I2.8). They fix the values of $g$ and $s$,

$$
g=s k \quad s=\frac{1}{\sqrt{1-\alpha k}} \quad k \equiv \frac{1}{2}(\beta-\alpha)+\sqrt{1+\frac{1}{4}(\beta-\alpha)^{2}}
$$

in terms of $\alpha$ and $\beta$. The remaining two conditions read

$$
\begin{aligned}
r s & =-\mathcal{E} \\
\epsilon_{0} & =\frac{1}{2}\left(g s-r^{2}\right) .
\end{aligned}
$$

Equation (2.12) provides the value of $r$,

$$
r=-\mathcal{E} \sqrt{1-\alpha k}
$$

while equation (2.13) leads to the value of the factorization energy in terms of the three parameters $\alpha, \beta, \mathcal{E}$.

Having proved that the Hamiltonian $h$ is factorizable, let us now consider a hierarchy of Hamiltonians

$$
h_{i}=B^{+}\left(g_{i}, s_{i}, r_{i}\right) B^{-}\left(g_{i}, s_{i}, r_{i}\right)+\sum_{j=0}^{i} \epsilon_{j} \quad i=0,1,2, \ldots
$$

whose first member $h_{0}$ coincides with $h$. Here $g_{i}, s_{i}, \epsilon_{i}, r_{i}$ are some parameters, the first three being assumed positive, and $g_{0}=g, s_{0}=s, r_{0}=r$.

Proceeding as in I, let us impose the shape invariance condition

$$
B^{-}\left(g_{i}, s_{i}, r_{i}\right) B^{+}\left(g_{i}, s_{i}, r_{i}\right)=B^{+}\left(g_{i+1}, s_{i+1}, r_{i+1}\right) B^{-}\left(g_{i+1}, s_{i+1}, r_{i+1}\right)+\epsilon_{i+1}
$$

where $i=0,1,2, \ldots$ It is equivalent to a set of four relations, of which the first two are again independent of $\mathcal{E}$ and coincide with equations (I2.15) and (I2.16), respectively. In I, the latter have been solved by introducing some new combinations of parameters

$$
\begin{aligned}
& u_{i}=g_{i}+\gamma s_{i} \quad v_{i}=g_{i}-\gamma s_{i} \quad \gamma \equiv \sqrt{\frac{\beta}{\alpha}} \\
& d_{i}=u_{i} v_{i} \quad t_{i}=\frac{v_{i}}{u_{i}}
\end{aligned}
$$

thereby leading to

$$
d_{i}=d \quad t_{i}=q^{-i} t \quad \text { or } \quad u_{i}=q^{i / 2} u \quad v_{i}=q^{-i / 2} v
$$


where

$$
d \equiv u v \quad t \equiv \frac{v}{u} \quad q \equiv \frac{1+\sqrt{\alpha \beta}}{1-\sqrt{\alpha \beta}} .
$$

The remaining two relations read

$$
\begin{aligned}
r_{i+1} s_{i+1} & =r_{i} s_{i} \\
\epsilon_{i+1} & =\frac{1}{2}\left(g_{i} s_{i}+g_{i+1} s_{i+1}+r_{i}^{2}-r_{i+1}^{2}\right) .
\end{aligned}
$$

On taking equations (2.12), (2.17), (2.19), and (2.20) into account, we obtain from (2.21) the solution for $r_{i}$,

$$
r_{i}=-\frac{2 \gamma \mathcal{E}}{u} q^{-i / 2}\left(1-\frac{t}{q^{i}}\right)^{-1} .
$$

Furthermore, equation (2.22) leads to the eigenvalues $e_{n}$ of $h$,

$$
e_{n}(q, t, \mathcal{E})=\sum_{i=0}^{n} \epsilon_{i}=\sum_{i=0}^{n-1} g_{i} s_{i}+\frac{1}{2} g_{n} s_{n}-\frac{1}{2} r_{n}^{2}=e_{n}(q, t, 0)+\Delta e_{n}(q, t, \mathcal{E})
$$

where

$$
e_{n}(q, t, 0)=\frac{K^{2}}{q+1}\left\{\left(1-\frac{t^{2}}{q^{n-1}}\right)[n]_{q}+\frac{1}{2}\left(q^{n}-\frac{t^{2}}{q^{n}}\right)\right\} \quad K \equiv u \sqrt{\frac{q+1}{4 \gamma}} \quad[n]_{q} \equiv \frac{q^{n}-1}{q-1}
$$

are the eigenvalues in the absence of electric field, given in (I2.30), and

$$
\Delta e_{n}(q, t, \mathcal{E})=-\frac{2 \gamma^{2} \mathcal{E}^{2}}{u^{2}} q^{-n}\left(1-\frac{t}{q^{n}}\right)^{-2}
$$

are the corrections due to the electric field. The latter can be rewritten as

$$
\Delta e_{n}(q, t, z)=-\frac{1}{2} K^{2} z^{2}(1-t)^{2} q^{-n}\left(1-\frac{t}{q^{n}}\right)^{-2}
$$

in terms of a new parameter proportional to $\mathcal{E}$,

$$
z \equiv 4 \gamma \sqrt{\frac{\gamma}{q+1}} \frac{\mathcal{E}}{u^{2}(1-t)}
$$

which will subsequently prove convenient (see equations (2.47) and (2.57)).

In contrast with conventional quantum mechanics, the additional negative contributions $\Delta e_{n}(q, t, z)$ are $n$ dependent for generic $\alpha$ and $\beta$ values. Since $q>1$, their absolute value decreases from $\frac{1}{2} K^{2} z^{2}$ to 0 when $n$ goes from 0 to $\infty$. As it can be inferred from the definition (2.4) of $h$, where the electric field breaks the symmetry under exchange of $X$ and 
$P, \Delta e_{n}(q, t, z)$ is not invariant under exchange of $\alpha$ and $\beta$ as it is the case for $e_{n}(q, t, 0)$. This will lead to different limiting cases in the next subsection.

The corrections to the excitation energies in the absence of electric field

$$
e_{n}(q, t, 0)-e_{0}(q, t, 0)=\frac{1}{2} K^{2}\left(1-\frac{t^{2}}{q^{n}}\right)[n]_{q}
$$

are given by

$$
\Delta e_{n}(q, t, z)-\Delta e_{0}(q, t, z)=\frac{1}{2}(q-1) K^{2} z^{2} q^{-n}\left(1-\frac{t^{2}}{q^{n}}\right)\left(1-\frac{t}{q^{n}}\right)^{-2}[n]_{q} .
$$

On taking the results for $g_{i}, s_{i}, r_{i}$, and $\epsilon_{i}$ into account, the Hamiltonians (2.15) of the SUSYQM hierarchy can be written as

$$
h_{i}=\frac{1}{2}\left(a_{i} P^{2}+b_{i} X^{2}\right)-\mathcal{E} X+c_{i} \quad i=0,1,2, \ldots
$$

where $a_{i}, b_{i}, c_{i}$ are constants independent of $\mathcal{E}$ and given by equation (I2.36). Going back to variables with dimensions, we get

$$
H_{i} \equiv \hbar \omega h_{i}=\frac{p^{2}}{2 m_{i}}+\frac{1}{2} m_{i} \omega_{i}^{2} x^{2}-\bar{q} \overline{\mathcal{E}} x+c_{i} \hbar \omega \quad m_{i}=\frac{m}{a_{i}} \quad \omega_{i}=\sqrt{a_{i} b_{i}} \omega .
$$

Note that contrary to the mass and the frequency, the electric field is the same for all the partners.

\subsection{Some special cases}

\subsubsection{Limit $\alpha \rightarrow 0$}

For small $\alpha$ values, we obtain

$$
q^{-n} \simeq 1-2 n \sqrt{\alpha \beta}+O(\alpha) \quad \frac{u}{\gamma}\left(1-\frac{t}{q^{n}}\right) \simeq 2 s(1-n \sqrt{\alpha \beta})+O(\alpha) .
$$

Inserting such results in (2.26) transforms the latter into

$$
\Delta e_{n} \simeq-\frac{\mathcal{E}^{2}}{2 s^{2}}+O(\alpha) \quad \text { where } \quad s \simeq 1+O(\alpha) .
$$

In the limit $\alpha \rightarrow 0$, we therefore get

$$
\Delta e_{n}(\mathcal{E})=-\frac{1}{2} \mathcal{E}^{2}
$$

which is independent of $n$ and of $\beta$ and is actually the same as in conventional quantum mechanics. We conclude that the $n$-dependent corrections to an exponential spectrum obtained in the general case reduce to $n$-independent corrections to a quadratic spectrum in the special case $\alpha=0$. Such a result would also follow from considering equations (2.7) and (2.8) for $\alpha=0$. 


\subsubsection{Limit $\beta \rightarrow 0$}

For small $\beta$ values, we obtain

$$
q^{-n} \simeq 1+2 n \sqrt{\alpha \beta}+O(\beta) \quad \frac{u}{\gamma}\left(1-\frac{t}{q^{n}}\right) \simeq 2(s+n g \alpha)+O(\sqrt{\beta})
$$

so that equation (2.26) now becomes

$$
\Delta e_{n} \simeq-\frac{\mathcal{E}^{2}}{2(s+n g \alpha)^{2}}+O(\sqrt{\beta})
$$

where

$$
g \simeq 1+O(\beta) \quad s \simeq \frac{1}{2} \alpha+\sqrt{1+\frac{1}{4} \alpha^{2}}+O(\beta) .
$$

In the limit $\beta \rightarrow 0$, we therefore get corrections

$$
\Delta e_{n}(\alpha, \mathcal{E})=-\frac{1}{2} \mathcal{E}^{2}\left[\left(n+\frac{1}{2}\right) \alpha+\sqrt{1+\frac{1}{4} \alpha^{2}}\right]^{-2}
$$

to a quadratic spectrum $e_{n}(\alpha)$. The latter can be obtained from equation (I3.6) by substituting $\alpha$ for $\beta$. We conclude that the essential feature of the general case, namely the $n$ dependence of the correction terms, is already present when there is only a nonzero minimal uncertainty in momentum.

The result (2.39) may also be derived directly from SUSYQM and shape invariance without resorting to a limiting procedure. Going back to the factorization and shape invariance conditions given in section 2.1 and setting $\beta=0$ therein, we are only left with two parameters $s_{i}$ and $r_{i}$, satisfying the conditions $s_{i+1}\left(s_{i+1}-\alpha\right)=s_{i}\left(s_{i}+\alpha\right), r_{i+1}=r_{i} s_{i} / s_{i+1}$, since $g_{i}=g=1$. This leads to $s_{i}=s+i \alpha$ and $r_{i}=-\mathcal{E} / s_{i}$, from which equation (2.39) can be easily obtained. The fact that $r_{i}$ is not independent of $i$, contrary to what happens for $\alpha=0$, is clearly responsible for the $n$ dependence of $\Delta e_{n}(\alpha, \mathcal{E})$ shown in $(2.39)$.

\subsubsection{Case $\alpha=\beta \neq 0$}

In I, we showed that whenever the two dimensionless deforming parameters $\alpha$ and $\beta$ are equal (which means that there is a specific relation $\bar{\alpha}=m^{2} \omega^{2} \bar{\beta}$ between the original parameters $\bar{\alpha}, \bar{\beta}$ of equation (2.3)), the harmonic oscillator Hamiltonian $\frac{1}{2}\left(P^{2}+X^{2}\right)$ reduces to a $q$-deformed harmonic oscillator one

$$
h_{\mathrm{osc}}=\frac{1}{4}(q+1)\left\{b, b^{+}\right\}
$$


where

$$
b^{+}=\frac{1}{\sqrt{q+1}}(X-\mathrm{i} P) \quad b=\frac{1}{\sqrt{q+1}}(X+\mathrm{i} P)
$$

satisfy the relation

$$
b b^{+}-q b^{+} b=I
$$

with $q=(1+\alpha) /(1-\alpha)$.

In terms of the operators (2.40) and (2.41), the Hamiltonian (2.4) can be rewritten as

$$
h=h_{\mathrm{osc}}-\frac{1}{2} \sqrt{q+1} \mathcal{E}\left(b^{+}+b\right) .
$$

Since for $\alpha=\beta$, we get $\gamma=1, t=0$ and $u=2 / \sqrt{1-\alpha}=\sqrt{2(q+1)}$, it follows from equation (2.26) or (2.27) that the correction term to the eigenvalues (I3.11) of $h_{\text {osc }}$ reads

$$
\Delta e_{n}(q, \mathcal{E})=-\frac{\mathcal{E}^{2}}{q+1} q^{-n}
$$

or

$$
\Delta e_{n}(q, z)=-\frac{1}{2} K^{2} z^{2} q^{-n} \quad K=\frac{1}{\sqrt{2}}(q+1) \quad z=2 \mathcal{E}(q+1)^{-3 / 2}
$$

which is again $n$ dependent.

\subsection{Hamiltonian eigenvectors}

Before going to the general case $0 \neq \alpha \neq \beta \neq 0$, it is worth considering the special case $\alpha=\beta \neq 0$, for which the Hamiltonian eigenvalues and eigenvectors depend only on two parameters instead of three.

\subsubsection{Case $\alpha=\beta \neq 0$}

In terms of the $q$-deformed boson creation and annihilation operators $b^{+}, b$, defined in equation (2.41), the operators $B^{ \pm}\left(g_{i}, s_{i}, r_{i}\right)$ corresponding to the Hamiltonian (2.15) of the SUSYQM hierarchy, assume the simple form

$$
B^{+}\left(q, z_{i}\right)=\frac{1}{\sqrt{2}} K q^{i / 2}\left(b^{+}-z_{i}\right) \quad B^{-}\left(q, z_{i}\right)=\frac{1}{\sqrt{2}} K q^{i / 2}\left(b-z_{i}\right)
$$

with

$$
z_{i} \equiv z q^{-i}
$$


In the ( $q$-deformed) Bargmann representation of the operators $b^{+}, b$, associated with the corresponding $q$-deformed coherent states [26, 27], they are represented by

$$
\mathcal{B}^{+}\left(q, z_{i}\right)=\frac{1}{\sqrt{2}} K q^{i / 2}\left(\xi-z_{i}\right) \quad \mathcal{B}^{-}\left(q, z_{i}\right)=\frac{1}{\sqrt{2}} K q^{i / 2}\left(\mathcal{D}_{q}-z_{i}\right)
$$

where $\xi$ is a complex variable and $\mathcal{D}_{q}$ is the $q$-differential operator defined by $\mathcal{D}_{q} \psi(\xi)=$ $[\psi(q \xi)-\psi(\xi)] /[(q-1) \xi]$.

Here it should be stressed that although the electric field remains constant in the Hamiltonians $h_{i}$ of the SUSYQM hierarchy, the corresponding parameter $z_{i}$ in the first-order operators $B^{ \pm}\left(q, z_{i}\right)$, involved in the factorization of $h_{i}$, is $i$ dependent, as shown in (2.47).

The ground state of $h$ is represented by a function $\psi_{0}(q, z ; \xi)$, which is a normalized solution of the equation

$$
\mathcal{B}^{-}(q, z) \psi_{0}(q, z ; \xi)=0 \quad \text { or } \quad \mathcal{D}_{q} \psi_{0}(q, z ; \xi)=z \psi_{0}(q, z ; \xi) .
$$

It is therefore the Bargmann representation of a $q$-deformed CS $|z\rangle_{q}$ with real $z$ and it is given by $[27]$

$$
\psi_{0}(q, z ; \xi)=\mathcal{N}_{0}(q, z) E_{q}(z \xi) \quad \mathcal{N}_{0}(q, z)=\left[E_{q}\left(|z|^{2}\right)\right]^{-1 / 2}
$$

where $E_{q}(\xi)=\sum_{n=0}^{\infty} \xi^{n} /[n]_{q}$ ! is a $q$-exponential.

The normalized excited state Bargmann wave functions can be determined recursively through the equations

$$
\begin{aligned}
\psi_{n+1}(q, z ; \xi) & =\left[e_{n+1}(q, z)-e_{0}(q, z)\right]^{-1 / 2} \mathcal{B}^{+}(q, z) \psi_{n}\left(q, z_{1} ; \xi\right) \\
& =\left\{[n+1]_{q}\left(1+\frac{(q-1) z^{2}}{q^{n+1}}\right)\right\}^{-1 / 2}(\xi-z) \psi_{n}\left(q, \frac{z}{q} ; \xi\right)
\end{aligned}
$$

where $n=0,1,2, \ldots$ It is straightforward to show that they are given by

$$
\psi_{n}(q, z ; \xi)=\mathcal{N}_{n}(q, z) \prod_{k=0}^{n-1}\left(\xi-\frac{z}{q^{k}}\right) E_{q}\left(\frac{z}{q^{n}} \xi\right)
$$

where

$$
\mathcal{N}_{n}(q, z)=\left\{[n]_{q} !\left(q^{-2 n+1}(1-q) z^{2} ; q\right)_{n}\right\}^{-1 / 2} \mathcal{N}_{0}\left(q, \frac{z}{q^{n}}\right)
$$

and $n=1,2, \ldots$ In $(2.53)$, the symbol $(a ; q)_{n}$ is defined as in equation (IA.5).

For $z \rightarrow 0$, the wave functions $\psi_{n}(q, z ; \xi)$ reduce to the Bargmann representation $\varphi_{n}(q ; \xi)=\xi^{n} / \sqrt{[n]_{q} !}$ of the $n$-q-boson states $|n\rangle_{q}=\left(b^{+}\right)^{n} / \sqrt{[n]_{q} !}|0\rangle_{q}$, which are the eigenvectors of $h_{\mathrm{osc}}$. 


\subsubsection{General case $0 \neq \alpha \neq \beta \neq 0$}

In the general case of unequal parameters $\alpha$ and $\beta, q$-boson creation and annihilation operators satisfying equation (2.42) are defined by

$$
b^{+}=\frac{1}{\sqrt{q+1}}\left(\frac{1}{\sqrt{\gamma}} X-\mathrm{i} \sqrt{\gamma} P\right) \quad b=\frac{1}{\sqrt{q+1}}\left(\frac{1}{\sqrt{\gamma}} X+\mathrm{i} \sqrt{\gamma} P\right) .
$$

The operators $B^{ \pm}\left(g_{i}, s_{i}, r_{i}\right)$, corresponding to $h_{i}$, can be written in terms of them as

$$
B^{+}\left(q, t_{i}, z_{i}\right)=\frac{1}{\sqrt{2}} K q^{i / 2}\left(b^{+}-t_{i} b-z_{i}\right) \quad B^{-}\left(q, t_{i}, z_{i}\right)=\frac{1}{\sqrt{2}} K q^{i / 2}\left(b-t_{i} b^{+}-z_{i}\right)
$$

and are represented by

$$
\mathcal{B}^{+}\left(q, t_{i}, z_{i}\right)=\frac{1}{\sqrt{2}} K q^{i / 2}\left(\xi-t_{i} \mathcal{D}_{q}-z_{i}\right) \quad \mathcal{B}^{-}\left(q, t_{i}, z_{i}\right)=\frac{1}{\sqrt{2}} K q^{i / 2}\left(\mathcal{D}_{q}-t_{i} \xi-z_{i}\right)
$$

in Bargmann representation. In equations (2.55) and (2.56), $z_{i}$ is given by

$$
z_{i}=-\frac{r_{i}}{K q^{i / 2}}=z q^{-i}(1-t)\left(1-\frac{t}{q^{i}}\right)^{-1}
$$

and reduces to the value given in (2.47) for $\alpha=\beta$ or $t=0$.

The ground state Bargmann wave function now satisfies the first-order difference equation

$$
\mathcal{D}_{q} \psi_{0}(q, t, z ; \xi)=(t \xi+z) \psi_{0}(q, t, z ; \xi)
$$

From the latter, it is clear that for large values of $|\xi|$, the behaviour of $\psi_{0}(q, t, z ; \xi)$ is the same as that of $\psi_{0}(q, t ; \xi)$, corresponding to $\mathcal{E}=0$. It therefore follows from I that equation (2.58) has a normalizable solution on the whole complex plane. By using properties (IA.2) and (IA.8) of the $q$-exponential and the $q$-differential operator, respectively, the latter can be written as

$$
\psi_{0}(q, t, z ; \xi)=\mathcal{N}_{0}(q, t, z) E_{q}(\lambda \xi) E_{q}(\mu \xi)
$$

where

$$
\lambda=\frac{1}{2} z+\Delta \quad \mu=\frac{1}{2} z-\Delta \quad \Delta=\sqrt{\frac{1}{4} z^{2}-\frac{t}{q-1}}
$$

and $\mathcal{N}_{0}(q, t, z)$ is some normalization coefficient.

On expanding the two $q$-exponentials in $(2.59), \psi_{0}(q, t, z ; \xi)$ becomes

$$
\psi_{0}(q, t, z ; \xi)=\mathcal{N}_{0}(q, t, z) \sum_{n=0}^{\infty} c_{n}(q, t, z) \varphi_{n}(q ; \xi)
$$


where

$$
\begin{aligned}
c_{n}(q, t, z)= & \frac{1}{\sqrt{[n]_{q} !}} \sum_{m=0}^{n}\left[\begin{array}{c}
n \\
m
\end{array}\right]_{q} \lambda^{m} \mu^{n-m}=\frac{1}{\sqrt{[n]_{q} !}} \sum_{p=0}^{[n / 2]} a_{n, p}(q) z^{n-2 p} t^{p} \\
a_{n, 0}(q)= & 1 \\
a_{n, p}(q)= & \sum_{r_{1}=2 p-1}^{n-1}\left[r_{1}\right]_{q} \sum_{r_{2}=2 p-3}^{r_{1}-2}\left[r_{2}\right]_{q} \ldots \sum_{r_{i}=2 p-2 i+1}^{r_{i-1}-2}\left[r_{i}\right]_{q} \cdots \sum_{r_{p}=1}^{r_{p-1}-2}\left[r_{p}\right]_{q} \\
& p=1,2, \ldots,[n / 2] \\
\mathcal{N}_{0}(q, t, z)= & \left(\sum_{n=0}^{\infty} c_{n}^{2}(q, t, z)\right)^{-1 / 2} .
\end{aligned}
$$

Here $[n / 2]$ denotes the largest integer contained in $n / 2$ and $\left[\begin{array}{c}n \\ m\end{array}\right]_{q}=[n]_{q} ! /\left([m]_{q} ![n-m]_{q} !\right)$ is a $q$-binomial coefficient. The proof of equation (2.64) is by induction over $p$.

Note that $\Delta$, defined in (2.60), may be real or imaginary according to the relative values of the parameters. For instance, for $\alpha=\beta$, we get $t=0$ and $\Delta=z / 2$ so that $\lambda=z$ and $\mu=0$ are real. In such a case, equation (2.59) reduces to equation (2.50). On the contrary, for $\mathcal{E}=0$, we get $z=0$ and $\Delta=\mathrm{i} \sqrt{t /(q-1)}$ so that $\lambda=\mathrm{i} \sqrt{t /(q-1)}$ and $\mu=\lambda^{*}$ are imaginary. Equation (2.59) then becomes

$$
\psi_{0}(q, t ; \xi)=\mathcal{N}_{0}(q, t) E_{q}\left(\mathrm{i} \sqrt{\frac{t}{q-1}} \xi\right) E_{q}\left(-\mathrm{i} \sqrt{\frac{t}{q-1}} \xi\right)=\mathcal{N}_{0}(q, t) E_{q^{2}}\left(\frac{t}{q+1} \xi^{2}\right)
$$

where in the last step we used a well-known property of the $q$-exponential [28, 29]. Equation (2.66) coincides with equation (I4.12).

The normalized excited state Bargmann wave functions satisfy the recursion relation

$$
\begin{aligned}
\psi_{n+1}(q, t, z ; \xi)= & \left\{[n+1]_{q}\left(1-\frac{t^{2}}{q^{n+1}}\right)\left[1+(q-1) z^{2} q^{-n-1}\left(1-\frac{t}{q^{n+1}}\right)^{-2}\right]\right\}^{-1 / 2} \\
& \times\left(\xi-t \mathcal{D}_{q}-z\right) \psi_{n}\left(q, t_{1}, z_{1} ; \xi\right) \quad n=0,1,2, \ldots
\end{aligned}
$$

where $t_{1}=t / q$ and $z_{1}=(z / q)(1-t)(1-t / q)^{-1}$.

The solution of equation (2.67) can be written as

$$
\psi_{n}(q, t, z ; \xi)=\mathcal{N}_{n}(q, t, z) P_{n}(q, t, z ; \xi) E_{q}\left(\lambda_{n} \xi\right) E_{q}\left(\mu_{n} \xi\right)
$$

where $\lambda_{n}$ and $\mu_{n}$ are given by equation (2.60) with $t$ and $z$ replaced by $t_{n}$ and $z_{n}$, respectively, $P_{n}(q, t, z ; \xi)$ is an $n$ th-degree polynomial in $\xi$, satisfying the relation

$$
P_{n+1}(q, t, z ; \xi)=(\xi-z) P_{n}\left(q, t_{1}, z_{1} ; \xi\right)-t\left(t_{n+1} \xi+z_{n+1}\right) P_{n}\left(q, t_{1}, z_{1} ; q \xi\right)-t \mathcal{D}_{q} P_{n}\left(q, t_{1}, z_{1} ; \xi\right)
$$


with $P_{0}(q, t, z ; \xi) \equiv 1$, and $\mathcal{N}_{n}(q, t, z)$ is a normalization coefficient fulfilling the recursion relation

$$
\begin{aligned}
\mathcal{N}_{n+1}(q, t, z)= & \left\{[n+1]_{q}\left(1-\frac{t^{2}}{q^{n+1}}\right)\left[1+(q-1) z^{2} q^{-n-1}\left(1-\frac{t}{q^{n+1}}\right)^{-2}\right]\right\}^{-1 / 2} \\
& \times \mathcal{N}_{n}\left(q, t_{1}, z_{1}\right) .
\end{aligned}
$$

It can be shown that for the first few $n$ values, the polynomials $P_{n}(q, t, z ; \xi)$ are given by

$$
\begin{aligned}
P_{1}(q, t, z ; \xi)= & \left(1-\frac{t^{2}}{q}\right)\left[\xi-z\left(1-\frac{t}{q}\right)^{-1}\right] \\
P_{2}(q, t, z ; \xi)= & \left(1-\frac{t^{2}}{q^{3}}\right)\left[\left(1-\frac{t^{2}}{q}\right) \xi^{2}-[2]_{q} \frac{z}{q}\left(1-\frac{t^{2}}{q}\right)\left(1-\frac{t}{q^{2}}\right)^{-1} \xi-t\right. \\
& \left.+\frac{z^{2}}{q}(1-t)\left(1+\frac{t}{q}\right)\left(1-\frac{t}{q^{2}}\right)^{-2}\right] \\
P_{3}(q, t, z ; \xi)= & \left(1-\frac{t^{2}}{q^{5}}\right)\left(1-\frac{t^{2}}{q^{3}}\right)\left\{\left(1-\frac{t^{2}}{q}\right) \xi^{3}-[3]_{q} \frac{z}{q^{2}}\left(1-\frac{t^{2}}{q}\right)\left(1-\frac{t}{q^{3}}\right)^{-1} \xi^{2}\right. \\
& +[3]_{q}\left[-\frac{t}{q}+\frac{z^{2}}{q^{3}}(1-t)\left(1+\frac{t}{q}\right)\left(1-\frac{t}{q^{3}}\right)^{-2}\right] \xi \\
& \left.+z \frac{t}{q^{2}}\left[\left([2]_{q}+q\right)-\left([2]_{q}+1\right) t\right]\left(1-\frac{t}{q}\right)\left(1-\frac{t}{q^{3}}\right)\right]^{-1} \\
& \left.-\frac{z^{3}}{q^{3}}(1-t)^{2}\left(1+\frac{t}{q^{2}}\right)\left[\left(1-\frac{t}{q}\right)\left(1-\frac{t}{q^{3}}\right)^{3}\right]^{-1}\right\}
\end{aligned}
$$

For $z=0$, they reduce to the corresponding polynomials obtained in (I4.26) - (I4.28), while for $t=0$, they give back the polynomials multiplying the $q$-exponential in equation (2.52), as it should be.

\subsection{Comparison with a previous work}

From equation (2.9) and equation (2.55) corresponding to $i=0$, it follows that the Hamiltonian $h$, defined in (2.4), is a Hermitian form bilinear in the $q$-boson creation and annihilation operators $b^{+}, b$, satisfying equation $(2.42)$ with $q>1$. The spectrum of the most general abstract Hamiltonian of such a type was investigated some years ago [25]. It is therefore interesting to see how our results compare with those previously obtained.

In [25], it has been shown that there exist two factorization schemes leading to two possible branches of the discrete spectrum for the considered Hamiltonian. In our case too, 
the existence of such schemes has been noticed: the parameter $k \equiv g / s$ indeed satisfies some quadratic equation, of which we have only kept the root with a plus sign in front of the radical, as shown in (2.11) (see also [14]). Our motivation for eliminating the other root with a minus sign in front of the radical has been purely physical. We indeed consider as previous authors working in the field of very small quadratic corrections to the canonical commutation relations leading to nonzero minimal uncertainties in position and momentum $[4,5,6,7,8,9,10,11,12,13]$ that our theory should give back the standard results when the deforming parameters $\alpha, \beta$ go to zero. The other solution for $k$ appears to be connected with a so-called classically singular representation (see e.g. [30]), which is regarded in such a theory as unphysical.

Focusing now our attention on the first factorization scheme of [25], we notice that our result for the energy spectrum, given in (2.24), (2.25) and (2.27), can be retrieved from equation (20) of [25] after identifying the parameters $\alpha_{0}, \beta_{0}$ and $\gamma_{0}$ of equation (23) in the same reference with $K / \sqrt{2},-K t / \sqrt{2}$ and $-K z / \sqrt{2}$, respectively.

In section 2.3, we have provided a thorough study of the corresponding Bargmann wave functions, which in contrast with what is claimed in [25] does not happen to be very simple. It indeed turns out that whilst the ground and first-excited states (see (2.59), (2.68) and (2.71)) are correctly given by equations (38) and (39) of that reference after identifying the parameters as above-mentioned and $z$ with $\xi$, such is not the case for the higher-excited states, which as immediately seen from (2.72) and (2.73), do not factorize as stated in [25]. Furthermore, our approach has directly led us to a compact expression for the ground-state wave function (2.59) as a product of two $q$-exponentials instead of the infinite product of quadratic factors displayed in [25]. The former can be easily expanded into the latter by employing well-known properties of $q$-exponentials [28, 29].

\section{D-dimensional harmonic oscillator}

Let us now consider the $D$-dimensional harmonic oscillator problem described by the Hamiltonian

$$
H=\frac{\boldsymbol{p}^{2}}{2 m}+\frac{1}{2} m \omega^{2} \boldsymbol{x}^{2}
$$

where the position and momentum components $x_{i}, p_{i}, i=1,2, \ldots, D$, satisfy modified commutation relations of the type $[6,8,9]$

$$
\left[x_{i}, p_{j}\right]=\mathrm{i} \hbar\left(\delta_{i, j}+\bar{\beta} \boldsymbol{p}^{2} \delta_{i, j}+\bar{\beta}^{\prime} p_{i} p_{j}\right)
$$




$$
\begin{aligned}
{\left[p_{i}, p_{j}\right] } & =0 \\
{\left[x_{i}, x_{j}\right] } & =\mathrm{i} \hbar \frac{\left(2 \bar{\beta}-\bar{\beta}^{\prime}\right)+\left(2 \bar{\beta}+\bar{\beta}^{\prime}\right) \bar{\beta} \boldsymbol{p}^{2}}{1+\bar{\beta} \boldsymbol{p}^{2}}\left(p_{i} x_{j}-p_{j} x_{i}\right)
\end{aligned}
$$

where $\bar{\beta}, \bar{\beta}^{\prime} \geq 0$. Such commutation relations imply isotropic nonzero minimal uncertainties in the position coordinates $\Delta x_{0 i}=\Delta x_{0}=\hbar \sqrt{D \bar{\beta}+\bar{\beta}^{\prime}}$, but none in the momentum coordinates, which are simultaneously diagonalizable.

In the momentum representation, the operators $p_{i}$ become multiplicative operators, while the operators $x_{i}$ are realized as differential operators $\mathrm{i} \hbar\left[\left(1+\bar{\beta} p^{2}\right) \partial / \partial p_{i}+\bar{\beta}^{\prime} p_{i} p_{j} \partial / \partial p_{j}+\bar{\gamma} p_{i}\right]$. Here $\bar{\gamma}$ is an arbitrary constant, which does not appear in the commutation relations (3.2) and only affects the weight function in the scalar product in momentum space

$$
\langle f \mid g\rangle=\int \frac{d^{D} \boldsymbol{p}}{\left[1+\left(\bar{\beta}+\bar{\beta}^{\prime}\right) p^{2}\right]^{1-\alpha}} f^{*}(\boldsymbol{p}) g(\boldsymbol{p})
$$

where

$$
\alpha=\frac{\bar{\gamma}-\frac{1}{2}(D-1) \bar{\beta}^{\prime}}{\bar{\beta}+\bar{\beta}^{\prime}}
$$

Since the Hamiltonian (3.1) is rotationally invariant, its momentum space eigenfunctions can be expressed as a product of a D-dimensional spherical harmonics $Y_{l_{D-1} \cdots l_{2} l_{1}}(\Omega)$ and a radial wave function $R_{n l}(p)$ (where $l=l_{D-1}$ ). In terms of a dimensionless variable $P=p a / \hbar$ and dimensionless parameters $\beta=\bar{\beta} \hbar^{2} / a^{2}, \beta^{\prime}=\bar{\beta}^{\prime} \hbar^{2} / a^{2}, \gamma=\bar{\gamma} \hbar^{2} / a^{2}$, where $a=\sqrt{\hbar /(m \omega)}$, the radial differential equation $[9]$ can be written as

$$
\begin{aligned}
& \frac{1}{2}\left\{-\left(f(P) \frac{d}{d P}\right)^{2}-\left[\frac{D-1}{P}+((D-1) \beta+2 \gamma) P\right] f(P) \frac{d}{d P}+\frac{L^{2}}{P^{2}}-\left(D \gamma-2 \beta L^{2}\right)\right. \\
& \left.+\left[1+\beta^{2} L^{2}-\gamma\left(D \beta+\beta^{\prime}+\gamma\right)\right] P^{2}\right\} R_{n l}(P)=e_{n l} R_{n l}(P)
\end{aligned}
$$

where $L^{2}$ is the eigenvalue of the square of the $D$-dimensional angular momentum

$$
L^{2}=l(l+D-2) \quad l=0,1,2, \ldots
$$

$n$ is the radial quantum number and

$$
f(P)=1+\beta_{0} P^{2} \quad \beta_{0}=\beta+\beta^{\prime} \quad e_{n l}=\frac{E_{n l}}{\hbar \omega} .
$$


The first-order derivative in (3.5) can be eliminated by setting

$$
R_{n l}(P)=P^{-(D-1) / 2}[f(P)]^{-\alpha / 2} \chi_{n l}(P) .
$$

The resulting equation reads

$$
h^{(l)} \chi_{n l}(P)=\tilde{e}_{n l} \chi_{n l}(P)
$$

where

$$
\begin{aligned}
& h^{(l)}=\frac{1}{2}\left\{-\left[f(P) \frac{d}{d P}\right]^{2}+\frac{a^{(l)}}{P^{2}}+b^{(l)} P^{2}\right\} \\
& a^{(l)}=L^{2}+\frac{1}{4}(D-3)(D-1)=\left(l+\frac{D-3}{2}\right)\left(l+\frac{D-1}{2}\right) \\
& b^{(l)}=1+\beta^{2}\left[L^{2}+\frac{1}{4}\left(D^{2}-1\right)\right]+\frac{1}{2}(D-1) \beta \beta^{\prime} \\
& \tilde{e}_{n l}=e_{n l}-\beta\left[L^{2}+\frac{1}{4}(D-1)^{2}\right]+\frac{1}{4}(D-1) \beta^{\prime} .
\end{aligned}
$$

From (3.8), it follows that in the space spanned by functions $\chi(P)$, the scalar product (3.3) becomes

$$
\left\langle\chi^{\prime} \mid \chi\right\rangle=\int_{0}^{\infty} \frac{d P}{f(P)} \chi^{\prime *}(P) \chi(P) .
$$

One should note that the arbitrary constant $\bar{\gamma}$, appearing in the momentum space realization of $x_{i}$ and in the scalar product (3.3), is absent from equations (3.9) - (3.14). From the very beginning, it is therefore obvious that neither $\tilde{e}_{n l}$ (hence $e_{n l}$ ) nor $\chi_{n l}(P)$ can depend on it.

\section{$3.1 \quad$ Energy spectrum}

Let us first prove that $h^{(l)}$ can be factorized as

$$
h^{(l)}=B^{+}(g, s) B^{-}(g, s)+\tilde{\epsilon}_{0}
$$

where

$$
B^{ \pm}(g, s)=\frac{1}{\sqrt{2}}\left(\mp f(P) \frac{d}{d P}+g P-\frac{s}{P}\right)
$$

and $\tilde{\epsilon}_{0}$ is the factorization energy. Here $g$ and $s$ are assumed to be two positive constants that are functions of $l$ and of the parameters $\beta, \beta^{\prime}$ of the problem. 
On inserting (3.16) in (3.15) and comparing the result with (3.10), we get the three conditions

$$
\begin{aligned}
s(s-1) & =a^{(l)} \\
g\left(g-\beta_{0}\right) & =b^{(l)} \\
\tilde{\epsilon}_{0} & =g s+\frac{1}{2}\left(g+\beta_{0} s\right) .
\end{aligned}
$$

Their solution is given by

$$
\begin{aligned}
s & =l+\frac{1}{2}(D-1) \\
g & =\frac{1}{2} \beta_{0}+\Delta^{(l)} \quad \Delta^{(l)}=\sqrt{1+\beta^{2} L^{2}+\frac{1}{4}\left(D \beta+\beta^{\prime}\right)^{2}} \\
\tilde{\epsilon}_{0} & =\frac{1}{2} \beta_{0}\left(2 l+D-\frac{1}{2}\right)+\left(l+\frac{D}{2}\right) \Delta^{(l)} .
\end{aligned}
$$

In the limit $\beta, \beta^{\prime} \rightarrow 0$, we get $s=l+\frac{1}{2}(D-1), g \rightarrow 1, \tilde{\epsilon}_{0} \rightarrow l+\frac{D}{2}$, which correspond to the usual factorization for the $D$-dimensional harmonic oscillator in conventional quantum mechanics (see, e.g., [31] for the three-dimensional case).

The next step consists in considering a hierarchy of Hamiltonians

$$
h_{i}^{(l)}=B^{+}\left(g_{i}, s_{i}\right) B^{-}\left(g_{i}, s_{i}\right)+\sum_{j=0}^{i} \tilde{\epsilon}_{j} \quad i=0,1,2, \ldots
$$

where $h_{0}^{(l)}=h^{(l)}, g_{0}=g, s_{0}=s$ and $g_{i}, s_{i}, i=1,2, \ldots$, are some positive constants. On imposing a shape invariance condition similar to equation (2.16), we obtain the set of three relations

$$
\begin{aligned}
s_{i+1}\left(s_{i+1}-1\right) & =s_{i}\left(s_{i}+1\right) \\
g_{i+1}\left(g_{i+1}-\beta_{0}\right) & =g_{i}\left(g_{i}+\beta_{0}\right) \\
\tilde{\epsilon}_{i+1} & =g_{i+1}\left(s_{i+1}+\frac{1}{2}\right)-g_{i}\left(s_{i}-\frac{1}{2}\right)+\frac{1}{2} \beta_{0}\left(s_{i+1}+s_{i}\right) .
\end{aligned}
$$

The solution of the first two is given by

$$
s_{i}=s+i \quad g_{i}=g+\beta_{0} i
$$

The third one, together with equation (3.19), leads to the eigenvalues

$$
\tilde{e}_{n}(g, s)=\sum_{i=0}^{n} \tilde{\epsilon}_{i}=g\left(2 n+s+\frac{1}{2}\right)+\beta_{0} s\left(2 n+\frac{1}{2}\right)+2 \beta_{0} n^{2} .
$$


Taking equations (3.20) and (3.21) into account, we can rewrite them as

$$
\tilde{e}_{n l}=\left(2 n+l+\frac{D}{2}\right) \Delta^{(l)}+\frac{1}{2} \beta_{0}\left(2 n+l+\frac{D}{2}\right)+\beta_{0}\left(l+\frac{D-1}{2}\right)\left(2 n+\frac{1}{2}\right)+2 \beta_{0} n^{2}
$$

or

$$
\tilde{e}_{N l}=\left(N+\frac{D}{2}\right) \Delta^{(l)}+\frac{1}{2} \beta_{0}\left(N^{2}+D N-L^{2}+D-\frac{1}{2}\right)
$$

in terms of $l$ and of either the radial quantum number $n$ or the principal quantum number $N=2 n+l$. Finally, from equation (3.13), it follows that the eigenvalues in the radial differential equation (3.5) can be expressed as

$$
e_{N l}=\left(N+\frac{D}{2}\right) \Delta^{(l)}+\frac{1}{2}\left[\left(\beta+\beta^{\prime}\right)\left(N+\frac{D}{2}\right)^{2}+\left(\beta-\beta^{\prime}\right)\left(L^{2}+\frac{D^{2}}{4}\right)+\beta^{\prime} \frac{D}{2}\right] \text {. }
$$

In the limit $\beta, \beta^{\prime} \rightarrow 0$, we obtain that $\tilde{e}_{N l}$ and $e_{N l}$ go to $e_{N}=N+\frac{D}{2}$, which is the conventional result. Equation (3.31) shows that for nonvanishing $\beta$, $\beta^{\prime}$, the spectrum of $H$, given by $E_{N l}=\hbar \omega e_{N l}$, is quadratic in $N$ with an additional $l$ dependence absent in the conventional case. The values obtained for $E_{N l}$ coincide with those given in equation (57) of $[9]$.

The results obtained for $g_{i}, s_{i}$ and $\tilde{\epsilon}_{i}$ allow us to write the Hamiltonians (3.23) of the SUSYQM hierarchy as

$$
h_{i}^{(l)}=\frac{1}{2}\left\{-\left[f(P) \frac{d}{d P}\right]^{2}+\frac{a_{i}^{(l)}}{P^{2}}+b_{i}^{(l)} P^{2}\right\}+c_{i}^{(l)}
$$

where

$$
\begin{aligned}
a_{i}^{(l)} & =\left(l+i+\frac{D-3}{2}\right)\left(l+i+\frac{D-1}{2}\right) \\
b_{i}^{(l)} & =b^{(l)}+2 \beta_{0} i \Delta^{(l)}+\beta_{0}^{2} i^{2} \\
c_{i}^{(l)} & =i\left[\beta_{0}\left(l+i-1+\frac{D}{2}\right)+\Delta^{(l)}\right] .
\end{aligned}
$$

In the limit $\beta, \beta^{\prime} \rightarrow 0, a_{i}^{(l)}$ remains unchanged while $b_{i}^{(l)} \rightarrow 1$ and $c_{i}^{(l)} \rightarrow i$.

As in conventional quantum mechanics, the supersymmetric partners coincide formally with some radial Hamiltonians corresponding to shifted $l$ values, $l+i$, where $i=1,2, \ldots$. It should be stressed that the angular part $Y_{l_{D-1} \cdots l_{2} l_{1}}(\Omega)$ of the wave functions being left unchanged, supersymmetry only concerns here the radial equation. 


\subsection{Radial wave functions}

In this subsection, we plan to determine the explicit form of the eigenfunctions $\chi_{n l}(P)$ of $h^{(l)}$. Since $\left(h^{(l)}\right)^{\dagger}=h^{(l)}$ with respect to scalar product (3.14), such eigenfunctions satisfy the orthonormality relation

$$
\int_{0}^{\infty} \frac{d P}{f(P)} \chi_{n^{\prime} l}^{*}(P) \chi_{n l}(P)=\delta_{n^{\prime}, n} .
$$

Note that with respect to (3.14), we also have $\left(B^{+}(g, s)\right)^{\dagger}=B^{-}(g, s)$.

The ground state wave function $\chi_{0 l}(P)=\chi_{0}(g, s ; P)$ of $h^{(l)}$ is obtained from the condition

$$
B^{-}(g, s) \chi_{0}(g, s ; P)=0
$$

and given by

$$
\chi_{0}(g, s ; P)=\mathcal{N}_{0}(g, s) P^{s}[f(P)]^{-\left(g+\beta_{0} s\right) /\left(2 \beta_{0}\right)}=\mathcal{N}_{0}(g, s) P^{\mu+\frac{1}{2}}[f(P)]^{-\frac{1}{2}(\lambda+\mu+1)} .
$$

Here $s$ and $g$ are given by equations (3.20) and (3.21), respectively, $\lambda$ and $\mu$ are defined by

$$
\lambda=\frac{1}{\beta_{0}}\left(g-\frac{1}{2} \beta_{0}\right) \quad \mu=s-\frac{1}{2}
$$

and the normalization coefficient is

$$
\mathcal{N}_{0 l}=\mathcal{N}_{0}(g, s)=\left(\frac{2 \Gamma(\lambda+\mu+2)}{\Gamma(\lambda+1) \Gamma(\mu+1)} \beta_{0}^{\mu+1}\right)^{1 / 2} .
$$

The excited state wave functions $\chi_{n, l}(P)=\chi_{n}(g, s ; P), n=1,2, \ldots$, can be obtained from the recursion relation

$$
\begin{aligned}
\chi_{n+1}(g, s ; P)= & {\left[\beta_{0}(n+1)(n+\lambda+\mu+2)\right]^{-1 / 2} \frac{1}{2}\left[-f(P) \frac{d}{d P}+\beta_{0}\left(\lambda+\frac{1}{2}\right) P-\frac{\mu+\frac{1}{2}}{P}\right] } \\
& \times \chi_{n}\left(g_{1}, s_{1} ; P\right) \quad n=0,1,2, \ldots
\end{aligned}
$$

where from (3.27) and (3.39), it follows that $g_{1}=g+\beta_{0}, s_{1}=s+1$ correspond to $\lambda_{1}=\lambda+1$, $\mu_{1}=\mu+1$.

Let us set

$$
\chi_{n}(g, s ; P)=\mathcal{N}_{n}(g, s) P_{n}^{(\lambda, \mu)}(z) P^{\mu+\frac{1}{2}}[f(P)]^{-\frac{1}{2}(\lambda+\mu+1)}
$$

where $\mathcal{N}_{n l}=\mathcal{N}_{n}(g, s)$ is some normalization coefficient and $P_{n}^{(\lambda, \mu)}(z)$ is some $(\lambda, \mu)$ dependent, $n$ th-degree polynomial in the variable

$$
z=\frac{\beta_{0} P^{2}-1}{1+\beta_{0} P^{2}}
$$


varying in the range $(-1,+1)$ (with $P_{0}^{(\lambda, \mu)}(z) \equiv 1$ ). Inserting $(3.42)$ in $(3.41)$ converts the latter into the relation

$$
\begin{aligned}
P_{n+1}^{(\lambda, \mu)}(z)= & {\left[\beta_{0}(n+1)(n+\lambda+\mu+2)\right]^{-1 / 2} \frac{\mathcal{N}_{n}\left(g_{1}, s_{1}\right)}{\mathcal{N}_{n+1}(g, s)} } \\
& \times \frac{1}{2}\left[-\left(1-z^{2}\right) \frac{d}{d z}+\lambda-\mu+(\lambda+\mu+2) z\right] P_{n}^{(\lambda+1, \mu+1)}(z) .
\end{aligned}
$$

The differential operator on the right-hand side of (3.44) can be recognized as the backward shift operator for Jacobi polynomials, satisfying the property [32]

$$
\left[-\left(1-z^{2}\right) \frac{d}{d z}+\lambda-\mu+(\lambda+\mu+2) z\right] P_{n}^{(\lambda+1, \mu+1)}(z)=2(n+1) P_{n+1}^{(\lambda, \mu)}(z) .
$$

Hence the polynomials of equation (3.42) can be identified with Jacobi polynomials, while $\mathcal{N}_{n}(g, s)$ satisfies the recursion relation

$$
\mathcal{N}_{n+1}(g, s)=\left(\frac{n+1}{\beta_{0}(n+\lambda+\mu+2)}\right)^{1 / 2} \mathcal{N}_{n}\left(g_{1}, s_{1}\right)
$$

from which we get

$$
\mathcal{N}_{n}(g, s)=\left(\frac{2(2 n+\lambda+\mu+1) n ! \Gamma(n+\lambda+\mu+1)}{\Gamma(n+\lambda+1) \Gamma(n+\mu+1)} \beta_{0}^{\mu+1}\right)^{1 / 2} .
$$

When combining equations (3.42) and (3.47) with (3.8), we finally obtain the same result as in equation (58) of [9].

\subsection{Alternative factorizations}

As a final point, we would like to comment on the factorization of $h^{(l)}$ carried out in section 3.1 .

Equation (3.23) and the corresponding shape invariance condition show that each element of the hierarchy $h_{i}^{(l)}, i=0,1,2, \ldots$, admits two different factorizations: this is an example of the so-called two-way factorization [33]. The first-order operators $B^{ \pm}\left(g_{i}, s_{i}\right)$ involved in the factorization act as shift operators, connecting pairs of eigenstates with the same energy, belonging to two consecutive Hamiltonians of the hierarchy.

The conventional harmonic oscillator radial equation is known to admit a four-way factorization [31]: there indeed exists another pair of factorizations associated with another hierarchy of Hamiltonians $h_{i}^{(l) \prime}, i=0,1,2, \ldots$ As before, the corresponding first-order 
operators $B^{ \pm \prime}\left(g_{i}, s_{i}\right)$ connect pairs of eigenstates with the same energy, belonging to two consecutive Hamiltonians of the second hierarchy. Since, however, the Hamiltonians of the two extended hierarchies ${ }^{1}$ are linked through the relation $h_{-i}^{(l) \prime}=h_{i}^{(l)}-2 i$, the shift operators of the second hierarchy also connect pairs of eigenstates with different energies, belonging to two consecutive Hamiltonians of the first hierarchy. For such a reason, one can combine both pairs of shift operators to construct ladder operators connecting the eigenstates of the same Hamiltonian [31] (see also [34]).

In the case of the deformed commutation relations (3.2), the harmonic oscillator radial equation (3.9) also admits a pair of alternative factorizations, where in the counterpart of (3.16) we choose $g>0$ and $s<0$ instead of both $g, s>0$. Distinguishing by primes all parameters relative to these alternative factorizations from those of section 3.1 , we obtain

$$
\begin{aligned}
s^{\prime} & =-l-\frac{1}{2}(D-3) \\
g^{\prime} & =\frac{1}{2} \beta_{0}+\Delta^{(l)} \\
\tilde{\epsilon}_{0}^{\prime} & =-\frac{1}{2} \beta_{0}\left(2 l+D-\frac{7}{2}\right)-\left(l+\frac{D-4}{2}\right) \Delta^{(l)}
\end{aligned}
$$

and

$$
\begin{aligned}
s_{i}^{\prime} & =s^{\prime}+i \\
g_{i}^{\prime} & =g^{\prime}+\beta_{0} i \\
\tilde{\epsilon}_{i}^{\prime} & =g_{i}^{\prime}\left(s_{i}^{\prime}+\frac{1}{2}\right)-g_{i-1}^{\prime}\left(s_{i-1}^{\prime}-\frac{1}{2}\right)+\frac{1}{2} \beta_{0}\left(s_{i}^{\prime}+s_{i-1}^{\prime}\right)
\end{aligned}
$$

for $i=1,2, \ldots$ For $\beta, \beta^{\prime} \rightarrow 0$, we get $s^{\prime}=-l-\frac{1}{2}(D-3), g^{\prime} \rightarrow 1, \tilde{\epsilon}_{0}^{\prime} \rightarrow-l-\frac{1}{2}(D-4)$ and $s_{i}^{\prime}=-l+i-\frac{1}{2}(D-3), g_{i}^{\prime} \rightarrow 1, \tilde{\epsilon}_{i}^{\prime} \rightarrow 2$, which agree with the conventional results [31].

Proceeding as in section 3.1, we can write the energy eigenvalues $\tilde{e}_{m}\left(g^{\prime}, s^{\prime}\right)=\sum_{i=0}^{m} \tilde{\epsilon}_{i}^{\prime}$ of $h^{(l)}$ as

$$
\begin{aligned}
\tilde{e}_{m l}= & \left(2 m-l-\frac{D-4}{2}\right) \Delta^{(l)}+\frac{1}{2} \beta_{0}\left(2 m-l-\frac{D-4}{2}\right)-\beta_{0}\left(l+\frac{D-3}{2}\right)\left(2 m+\frac{1}{2}\right) \\
& +2 \beta_{0} m^{2} .
\end{aligned}
$$

\footnotetext{
${ }^{1}$ By extended hierarchies, we mean the sets of Hamiltonians $h_{i}^{(l)}$ or $h_{i}^{(l) \prime}$ obtained by letting $i$ run over $\mathbf{Z}$. Some of these Hamiltonians may be unphysical. For instance, in the case of $h_{i}^{(l)}$ given in (3.32), unphysical Hamiltonians correspond to $i<-l$.
} 
The comparison with equations (3.29) and (3.30) shows that the new quantum number $m$ is related to the radial and principal quantum numbers, $n$ and $N$, through the relation

$$
m=n+l+\frac{1}{2}(D-2)=\frac{1}{2}(N+l+D-2) .
$$

We have therefore rederived the energy spectrum of $h^{(l)}$ in an alternative way.

The second Hamiltonian hierarchy $h_{i}^{(l) \prime}, i=0,1,2, \ldots$, containing $(3.10)$ as its first member, is given by an expression similar to (3.32) with

$$
\begin{aligned}
a_{i}^{(l) \prime} & =\left(l-i+\frac{D-3}{2}\right)\left(l-i+\frac{D-1}{2}\right) \\
b_{i}^{(l) \prime} & =b^{(l)}+2 \beta_{0} i \Delta^{(l)}+\beta_{0}^{2} i^{2} \\
c_{i}^{(l) \prime} & =i\left[\beta_{0}\left(-l+i+1-\frac{D}{2}\right)+\Delta^{(l)}\right] .
\end{aligned}
$$

For $\beta, \beta^{\prime} \rightarrow 0, a_{i}^{(l) \prime}$ remains unchanged, but $b_{i}^{(l) \prime} \rightarrow 1$ and $c_{i}^{(l) \prime} \rightarrow i$, so that we recover the property $h_{-i}^{(l) \prime}=h_{i}^{(l)}-2 i$ for the Hamiltonians of the extended hierarchies. For nonvanishing $\beta, \beta^{\prime}$, however, there is no simple relation between them and the only common member is $h^{(l)}=h_{0}^{(l)}=h_{0}^{(l) \prime}$. Ladder operators cannot therefore be built by combining the two different types of shift operators. Such ladder operators have actually been constructed by using another method [10] and they appear to be very complicated operators.

\section{Conclusion}

In the present paper, we have continued with the application, initiated in I, of combined factorization and shape invariance techniques to quantum mechanical problems in the context of a theory based on some deformed canonical commutation relations and predicting nonzero minimal uncertainties in position and/or momentum.

To start with, we have determined for the first time the spectrum and the eigenvectors of a one-dimensional harmonic oscillator in a uniform electric field $\mathcal{E}$. We have established that whenever $\alpha \neq 0$, i.e., whenever there is a nonzero minimal uncertainty in momentum, the correction to the harmonic oscillator eigenvalues due to the electric field depends on the energy level and actually decreases when going up in energy. This is true whether there is also a nonzero minimal uncertainty in position or no, i.e., whether $\beta \neq 0$ or $\beta=0$. As was shown in I, in the former case the harmonic oscillator spectrum is exponential, whereas in the latter it is quadratic. In contrast, whenever $\alpha=0$ and $\beta \neq 0$, the electric field 
induces a constant shift of the (quadratic) harmonic oscillator spectrum, as is the case in conventional quantum mechanics.

In addition, we have shown that although the electric field remains the same in all the supersymmetric partners of our original Hamiltonian, its effect on the eigenvectors is rather complicated. The latter have been fully determined for $\alpha=\beta \neq 0$, in which case the harmonic oscillator with nonzero minimal uncertainties in both position and momentum reduces to a $q$-deformed harmonic oscillator with $q>1$. In the general case $0 \neq \alpha \neq \beta \neq 0$, the ground state and the first few excited states have been given in terms of $n$-q-boson states. In both instances, use has been made of a ( $q$-deformed) Bargmann representation of the latter and of $q$-differential calculus.

Furthermore, we have shown how our results compare with those of a previous study of the spectrum of the most general Hermitian Hamiltonian that is bilinear in $q$-boson creation and annihilation operators with $q>1$.

In the second part of our paper, we have reconsidered the problem of a $D$-dimensional harmonic oscillator when there are isotropic nonzero minimal uncertainties in the position coordinates, depending on two parameters $\beta, \beta^{\prime}$. We have established that our SUSYQM techniques can be extended to deal with the corresponding radial equation in the momentum representation. As a result, we have rederived in a very simple way both the spectrum and the momentum radial wave functions, previously found through lengthy differential equation techniques [9]. Finally, we have commented on various factorizations of the radial Hamiltonian and stressed both the resemblances and the differences between the deformed case and the conventional one.

The second part of our paper opens the way to other yet unsolved $D$-dimensional problems. Among these, we may quote that of a $D$-dimensional harmonic oscillator in a magnetic field, which would be in line with the first part of our paper.

\section{Acknowledgments}

CQ is a Research Director of the National Fund for Scientific Research (FNRS), Belgium. VMT is very grateful to Professor C. Quesne for warm hospitality at Université Libre de Bruxelles and thanks the National Fund for Scientific Research (FNRS), Belgium, for financial support. 


\section{References}

[1] Gross D J and Mende P F 1988 Nucl. Phys. B 303407

[2] Maggiore M 1993 Phys. Lett. B 30465

[3] Witten E 1996 Phys. Today 4924

[4] Kempf A 1994 Quantum field theory with nonzero minimal uncertainties in position and momentum Preprint hep-th/9405067

[5] Kempf A 1994 J. Math. Phys. 354483

Hinrichsen H and Kempf A 1996 J. Math. Phys. 372121

[6] Kempf A 1997 J. Phys. A: Math. Gen. 302093

[7] Kempf A 2001 Phys. Rev. D 63083514

Kempf A and Niemeyer J C 2001 Phys. Rev. D 64103501

[8] Kempf A, Mangano G and Mann R B 1995 Phys. Rev. D 521108

[9] Chang L N, Minic D, Okamura N and Takeuchi T 2002 Phys. Rev. D 65125027

[10] Dadić I, Jonke L and Meljanac S 2003 Phys. Rev. D 67087701

[11] Brau F 1999 J. Phys. A: Math. Gen. 327691

[12] Akhoury R and Yao Y-P 2003 Phys. Lett. B 57237

[13] Kempf A 1993 J. Math. Phys. 34969

[14] Quesne C and Tkachuk V M 2003 J. Phys. A: Math. Gen. 3610373

[15] Cooper F, Khare A and Sukhatme U 1995 Phys. Rep. 251267

Cooper F, Khare A and Sukhatme U 2001 Supersymmetry in Quantum Mechanics (Singapore: World Scientific)

[16] Junker G 1996 Supersymmetric Methods in Quantum and Statistical Physics (Berlin: Springer) 
[17] Gendenshtein L E 1983 Pis'ma Zh. Eksp. Teor. Fiz. 38299 Gendenshtein L E 1983 JETP Lett. 38356 (Engl. Transl.)

[18] Dabrowska J, Khare A and Sukhatme U 1988 J. Phys. A: Math. Gen. 21 L195

[19] Schrödinger E 1940 Proc. R. Irish Acad. A 46 9, 183

Schrödinger E 1941 Proc. R. Irish Acad. A 4753

[20] Infeld L and Hull T E 1951 Rev. Mod. Phys. 2321

[21] Cariñena J F and Ramos A 2000 Rev. Math. Phys. 121279

[22] Spiridonov V 1992 Phys. Rev. Lett. 69398

Spiridonov V 1992 Mod. Phys. Lett. A 71241

[23] Khare A and Sukhatme U P 1993 J. Phys. A: Math. Gen. 26 L901

Barclay D T, Dutt R, Gangopadhyaya A, Khare A, Pagnamenta A and Sukhatme U 1993 Phys. Rev. A 482786

[24] Lutzenko I, Spiridonov V and Zhedanov A 1995 Phys. Lett. A 204236

[25] Loutsenko I, Spiridonov V, Vinet L and Zhedanov A 1998 J. Phys. A: Math. Gen. 31 9081

[26] Spiridonov V 1995 Phys. Rev. A 521909

Spiridonov V 1996 Phys. Rev. A 532903

[27] Quesne C, Penson K A and Tkachuk V M 2003 Phys. Lett. A 31329

[28] Ubriaco M R 1992 Phys. Lett. A 1631

[29] Quesne C 2003 Disentangling $q$-exponentials: A general approach Preprint math$\mathrm{ph} / 0310038$

[30] Aizawa N 1993 Phys. Lett. A 177195

[31] Fernández C D J, Negro J and del Olmo M A 1996 Ann. Phys., NY 252386 
[32] Koekoek R and Swarttouw R F 1994 The Askey-scheme of hypergeometric orthogonal polynomials and its $q$-analogue Report No 94-05 Delft University of Technology (Preprint math.CA/9602214)

[33] de Lange O L and Raab R E 1991 Operator Methods in Quantum Mechanics (Oxford: Clarendon)

[34] Del Sol Mesa A and Quesne C 2002 J. Phys. A: Math. Gen. 352857 